\title{
The Phenomenological Description of Plastic Flow in Solids
}

\author{
O. V. Yushchenko and D. S. Trotskaya \\ Sumy State University, 2 Rimskii-Korsakov Street, Sumy 40007, Ukraine \\ Correspondence should be addressed to O. V. Yushchenko; yushchenko@phe.sumdu.edu.ua
}

Received 30 April 2013; Accepted 29 August 2013

Academic Editor: Kazuhiko Endo

Copyright (C) 2013 O. V. Yushchenko and D. S. Trotskaya. This is an open access article distributed under the Creative Commons Attribution License, which permits unrestricted use, distribution, and reproduction in any medium, provided the original work is properly cited.

In the framework of the phenomenological scheme a self-consistent description of the transition from the solid state to the plastic flow was presented taking into account the point defects such as interstitials and vacancies. On the basis of the system of synergistic equations the dependencies of the internal stresses and curvature of the velocity profile of the shear displacement on the order parameter as well as the stationary distribution of the concentration of vacancies were found.

\section{Introduction}

The processes occurring during the deformation of solids have attracted attention for a long time [1]. Moreover it is well known that the elastic deformation vanishes after the removal of the applied stress, and plastic deformation is retained even after removal of all external influences. Exception is the viscous flow of the material, which can continue indefinitely even when exposed to an arbitrarily small external stress (and the flow rate increases with stress). According to [2] it turns that some properties (elasticity and viscosity) are not unique to solids, but they are inherent in continuous media (e.g., granular materials, supercooled liquid polymers, suspensions, some gels, emulsions, etc.). In this regard, the science of the deformation and flow of continuous media (rheology) has received a great development recently [2,3]. Particularly the plastic deformation should be noted: on the one hand, it is typical for many natural and technological processes and, on the other hand, it is difficult in terms of the theoretical description. Usually the plastic deformation of crystals is associated with the emergence and movement of the dislocations within the grains and can be implemented by slipping and twinning [1]. Although many methods are applied to the numerical simulation of the dynamics of dislocation lines [4-8], the description of the plastic deformation of crystals remains a difficult task. In the case of amorphous materials and continuous media the analytical description of the plastic flow is possible only within the framework of the phenomenological theories, where the material is considered as homogeneous. The purpose of this paper is to construct a self-consistent phenomenological scheme, which allows describing the transition of solids to the plastic flow state. Since the plastic flow always arises at the microscopic level, that is, at the level of elementary point defects of different physical nature, the influence of point defects on the behavior of solids under the action of the given stress will be an actual task. And although at the moment the theories, associated with the movements of linear defects, have received the increasing development, nevertheless, in some cases (e.g., the return of the metal at low temperatures from the state of strain hardening) only interstitials and vacancies will be mobile, in contrast to the dislocation.

In this paper we propose a phenomenological scheme, within framework of which the transition from a solid state to a plastic flow is presented as a self-organization process of the elementary particles of a material taking into account the ensemble of vacancies and the external influence. Section 2 discusses the main variables that characterize the state of the system. The self-consistent system of equations describing the behavior of a solid is presented in Section 3. The analysis of the system in the framework of the adiabatic approximation is carried out in Section 4. Stationary flow regime is studied in Section 5. 


\section{Concentration of Vacancies}

Let us consider the dynamics of the material in the case of shear strain, when the displacement is given by the function $\mathbf{u}(\mathbf{r}, t)$. Usually, solid density deviation $\delta \rho$ can be associated with the expression

$$
-\bar{\rho} \operatorname{div} \mathbf{u}=-\bar{\rho} u_{i i}
$$

where $\bar{\rho}$ is an average density of the material, $|\delta \rho| \ll \bar{\rho}$; $u_{i i}$ trace of the strain tensor [1].

The understanding of the basic laws of plastic flow can be achieved in the framework of the hydrodynamic theory $[9,10]$, where the auxiliary variable was introduced

$$
m \equiv \frac{\delta \rho}{\bar{\rho}}+u_{i i}
$$

The last one meets the local fraction of the free volume. On the other hand, the parameter $m$ for crystalline solids can be interpreted as the concentration of vacancies and is given by the equation

$$
m=\frac{n_{0}(\mathbf{r}, t)-n(\mathbf{r}, t)}{n_{0}(\mathbf{r}, t)}
$$

where $n_{0}(\mathbf{r}, t)$ is the density of lattice sites, which depends on both the temperature and the strain and $n(\mathbf{r}, t)$ is the number of particles per unit of material volume (it depends on the strain) [9].

In the case of amorphous solids the concept of the "lattice sites" and "vacancies" or "interstitial atom" is not so obvious, and it is necessary to give a more general interpretation of the density $n_{0}(\mathbf{r}, t)$. Further, we will base on the assumption that the amorphous sample must have a set of periodically arranged in the space positions ("sites"), in each of which the atom can be in local equilibrium for a long time.

For crystals $n_{0}$ could be treated as density of the particles, which would have a system under an infinite compression pressure at a fixed value of the lattice constant. Consequently for the amorphous solids parameter $n_{0}$ can be defined as the particle density under an infinite compression pressure at a constant distance to the nearest neighbors [10]. Of course, it is difficult to realize experimentally the compression at a constant distance to the nearest neighbors, but, the accurate estimate of $n_{0}$ can be obtained, if the compression is performed at constant temperature and the distance to the nearest neighbor is properly defined. As a result, to determine $n_{0}$ at a given pressure $P$ and temperature $T$ with reasonable accuracy one can use the approximation [10]

$$
n_{0}=f(P, T)=\lim _{P^{\prime} \rightarrow \infty}\left[\frac{r_{s}\left(P^{\prime}, T\right)}{r_{s}(P, T)}\right]^{3} n\left(P^{\prime}, T\right),
$$

where $n\left(P^{\prime}, T\right)$ is a density of particles at pressure $P^{\prime}$ and temperature $T$ and $r_{s}(P, T)$ is the distance between nearest neighbors, determined by X-ray or neutron diffraction.

It turns out that the value of $m$ will be critical for the description of the plastic deformation of solids. Indeed, in the absence of vacancies $\left(n=n_{0}, m=0\right)$ the material is in a solid state when the external stress gives rise to only elastic deformation; in the case of vacancies $\left(n<n_{0}, m \leqslant 1\right)$ we get the state when the external stress leads to plastic flow of the material.

\section{Synergetic System of Equations}

The dynamic equations for the density and momentum density in the deterministic case can be represented in a standard way $[8,11]$

$$
\begin{gathered}
\frac{\partial}{\partial t} \delta \rho=-\bar{\rho} \operatorname{div} \mathbf{v}, \\
\bar{\rho} \frac{\partial}{\partial t} \mathbf{v}=\nabla \sigma+\eta_{0} \nabla^{2} \mathbf{v},
\end{gathered}
$$

where $\mathbf{v}=\partial \mathbf{u} / \partial t$ is the rate of change of the shear displacement, $\sigma$ is the elastic stresses in the sample, $\eta_{0}$ is the dynamic viscosity of the material, and the operator $\nabla=\partial / \partial \mathbf{r}$. Equations (5)-(6) have the same form as the hydrodynamic equations for one-component fluids [12].

Using the definition of $m$, the relationships for the velocity $\mathbf{v}$ and stress $\sigma$

$$
\mathbf{v}=\lambda_{0}\left(\frac{\delta F}{\delta \mathbf{u}}\right)_{\rho}, \quad \nabla \sigma=-\bar{\rho} \nabla\left(\frac{\delta F}{\delta \rho}\right)_{\mathbf{u}}-\left(\frac{\delta F}{\delta \mathbf{u}}\right)_{\rho}
$$

with the free energy functional $F=F\{\rho, \mathbf{u}\}\left(\lambda_{0}\right.$ is a kinetic coefficient), and the identities

$$
\left(\frac{\delta}{\delta \mathbf{u}}\right)_{\rho}=\left(\frac{\delta}{\delta \mathbf{u}}\right)_{m}-\nabla\left(\frac{\delta}{\delta m}\right)_{\mathbf{u}}, \quad\left(\frac{\delta}{\delta m}\right)_{\mathbf{u}}=\bar{\rho}\left(\frac{\delta}{\delta \rho}\right)_{\mathbf{u}},
$$

one can obtain [13] the equation of motion for the parameter $m$

$$
\dot{m}=\lambda_{0} \nabla^{2} \sigma+\lambda_{0} \nabla^{2}\left(\frac{\delta F}{\delta m}\right)_{\mathbf{u}}
$$

instead of (5).

Parameter $m$ is convenient to be chosen as an order parameter, which distinguishes the solid state and the plastic flow state. Then, to find the variational derivative in (9) we write the free energy of the system in the form of the Landau expansion for the second order phase transition

$$
F(m)=\frac{A}{2} m^{2}+\frac{B}{4} m^{4},
$$

where $A, B$ are the Landau expansion coefficients. For further consideration we restrict the quadratic term in (10), taking $A>0$.

It should also be noted that in [13] the consideration of the corresponding transition is limited by the analysis of (6) and (9) supplemented by the stochastic components. In this case, the dependence of the shear modulus $G$ on the parameter $m$ is fundamental, when two approximations are using the expansion in powers of $m$ and the inverse exponential 
function. As a result, near the glass transition with decreasing parameter $m$ the shear modulus drops sharply from the final value $G_{0}$ to zero (value, which is characteristic for the liquid state). However, for the construction of an adequate analytical scheme according to the Ruelle-Takens theorem [14], a self-consistent behavior of three degrees of freedom should be considered. It is known that a simple threeparameter scheme, describing the self-organizing system is the Lorenz system $[15,16]$. As the study of the selforganization processes, occurring in the ensemble of particles [17] or in a solid [18], shows, it is necessary to take into account the characteristics of each system and the relationships of its basic parameters, except the use of the main approaches for the construction of Lorentz mathematical model.

Based on the above it is natural to assume that the third parameter characterizing the system under study is the field of elastic stresses $\sigma$. As a result the system of synergistic equations, describing the transition to a plastic flow state and taking into account dissipative terms, becomes

$$
\begin{gathered}
\dot{m}=-\frac{m}{t_{m}}+\lambda_{0} \nabla^{2} \sigma+\lambda_{0} A \nabla^{2} m+g_{m} \nu \nabla^{2} \mathbf{v}, \\
\dot{\mathbf{v}}=\frac{1}{\bar{\rho}} \nabla \sigma+\frac{\eta_{0}}{\bar{\rho}} \nabla^{2} \mathbf{v}-g_{v} m \sigma \\
\dot{\sigma}=\frac{\sigma_{e}-\sigma}{t_{\sigma}}-g_{\sigma} \nu m \nabla^{2} \mathbf{v}
\end{gathered}
$$

In contrast to the original relation (9) for the rate of change of the concentration of vacancies $m$ in (11) we have used two additional terms. The first of these is a microscopic dissipation channel with a characteristic relaxation time $t_{m}$ and the last one is the hydrodynamic contribution $\nu \nabla^{2} \mathbf{v}$, taking into account the spatial variation of the velocity of the shear displacement $\left(g_{m}>0\right.$ is the coupling coefficient, $\nu=\eta_{0} / \bar{\rho}$ is a kinematic viscosity). In (12) (comparing with (6)) the additional term is also present. It takes into account the microscopic dissipation due to the mutual action of shear stresses and the number of vacancies on the change in the rate of shear displacement ( $g_{v}-$ a positive constant). The velocity of shear displacement plays the role of the field conjugated to the order parameter $m$. The role of the control parameter in the system of synergetic (11)-(13) is played by the stress $\sigma$, for which the microscopic relaxation during time $t_{\sigma}$ to the value $\sigma_{e}$, given by the external influences, is taken into account and the relaxation due to the redistribution of vacancies by the spatial variation of the velocity of the shear displacement ( $g_{\sigma}$ is a positive coupling constant) is considered.

The system of (11)-(13) is very hard to research, because it has a large set of constants: the relaxation times $t_{m}, t_{\sigma}$, material parameters $\bar{\rho}, \lambda_{0}, \eta_{0}\left(\nu=\lambda_{0} / \bar{\rho}\right)$, the constant expansion of Landau $A$, voltage $\sigma_{e}$, and coupling constants $g_{i}, i=m, v, \sigma$. Therefore, to analyze the system (11)-(13) it is convenient to use dimensionless variables for the time coordinate (for simplicity restrict dimensional case), the order parameter, the conjugate field, and control parameter to the appropriate scale:

$$
\begin{gathered}
t_{m}, l=\left(t_{m} \lambda_{0} A\right)^{1 / 2}, \quad m_{S}=l\left(v \tau t_{\sigma} g_{v} g_{\sigma}\right)^{-1 / 2}, \\
v_{S}=\frac{\lambda_{0} A}{v g_{m}} \frac{l}{\sqrt{v \tau t_{\sigma} g_{v} g_{\sigma}}}, \quad \sigma_{S}=\frac{l^{2}}{v \tau t_{m} g_{v} g_{m}},
\end{gathered}
$$

where we have introduced the hydrodynamic time scale $\tau=$ $\eta_{0} / G$.

As a result of the behavior of solids under external loading with the ensemble of vacancies there is a dimensionless system of equations

$$
\begin{gathered}
\dot{m}=-m+\alpha \sigma^{\prime \prime}+m^{\prime \prime}+v^{\prime \prime}, \\
\frac{\tau}{t_{m}} \dot{v}=\beta \sigma^{\prime}+\gamma^{2} v^{\prime \prime}-m \sigma, \\
\frac{t_{\sigma}}{t_{m}} \dot{\sigma}=\sigma_{e}-\sigma-m v^{\prime \prime} .
\end{gathered}
$$

Here the prime denotes the coordinate, and the additional parameters characterizing the ratio of the constants and parameters of the material are given by

$$
\begin{aligned}
& \alpha=\left(\frac{\lambda_{0} t_{\sigma} g_{\sigma}}{A \nu \tau t_{m} g_{v} g_{m}^{2}}\right)^{1 / 2}, \\
& \beta=\frac{\left(\nu \tau t_{\sigma} g_{v} g_{\sigma}\right)^{1 / 2}}{\bar{\rho} g_{v} t_{m} \lambda_{0} A}, \\
& \gamma^{2}=\frac{\eta_{0} \tau}{\bar{\rho} l^{2}} .
\end{aligned}
$$

\section{The Adiabatic Approximation}

Despite a significant simplification of the system (15) compared with (11)-(13), it is still quite difficult to analyze, so you need to use the approximation. The latter two equations of the system are given by the time $\tau, t_{\sigma}$, and $t_{m}$. Take into account that the corresponding relaxation time of the vacancy concentration is sufficiently large and is far superior to the hydrodynamic time scale $\tau$ and the stress relaxation $t_{\sigma}$. As a result of dissipative systems for real picture of the transition from a solid state to a plastic state it is realized when

$$
\frac{\tau}{t_{m}} \ll 1 ; \quad \frac{t_{\sigma}}{t_{m}} \ll 1 .
$$

Since the rate of change of the dimensionless $\dot{m}, \dot{v}, \dot{\sigma}$ is of the same order of magnitude, then (17) allows us to neglect the left side of the last two equations of (15). Moreover, by analyzing the expression (16) in the adiabatic approximation (17) we can put $\alpha, \beta \ll 1$. This leads to the simplified system of equations

$$
\begin{gathered}
\dot{m}=-m+m^{\prime \prime}+v^{\prime \prime}, \\
0=\gamma^{2} v^{\prime \prime}-m \sigma, \\
0=\sigma_{e}-\sigma-m v^{\prime \prime},
\end{gathered}
$$




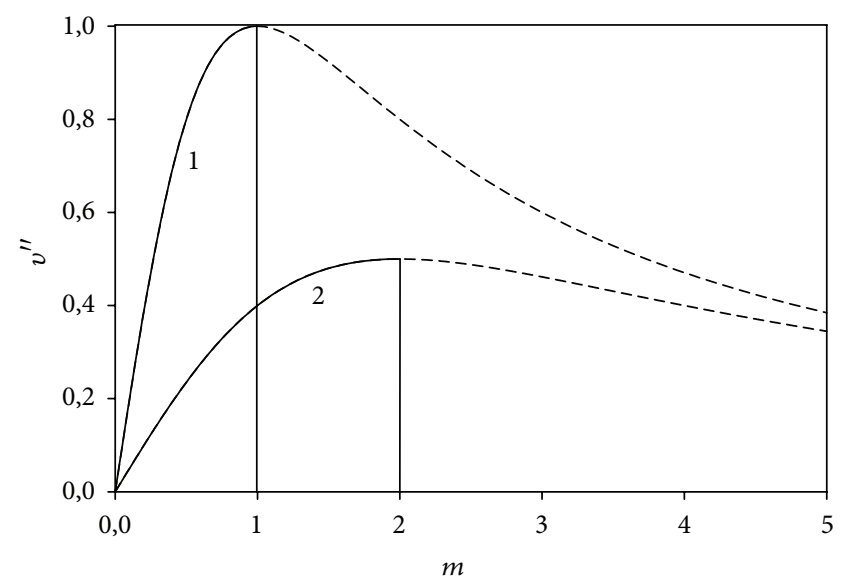

(a)

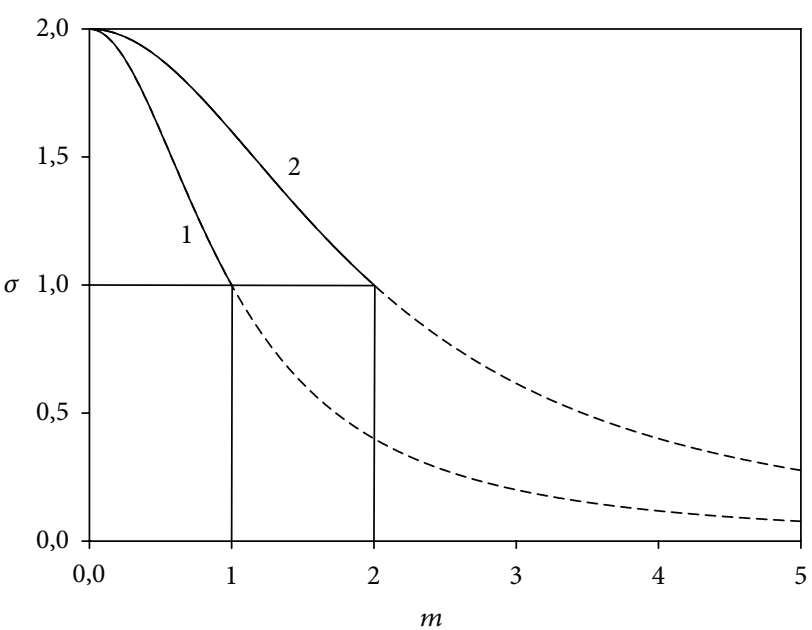

(b)

Figure 1: Dependence camber shift offset (a) and internal stresses (b): the concentration of vacancies in $\sigma_{e}=2 ; 1-\gamma=1,2-\gamma=2$.

from which we can obtain the dependence of the conjugate field and control parameter of the order parameter

$$
v^{\prime \prime}=\frac{m \sigma_{e}}{\gamma^{2}+m^{2}}, \quad \sigma=\frac{\gamma^{2} \sigma_{e}}{\gamma^{2}+m^{2}} .
$$

Thus, as shown in Figure 1(a) an increase in the vacancy concentration in the range bounded by the maximum value increases the curvature of the velocity of the shear displacement (dashed lines correspond to nonphysical area $m>\gamma$ ). In the case of internal stress $\sigma$ increase in concentration $m$ in the same interval leads to gradual relaxation of the value $\sigma_{e}$, given by external shocks to the value $\sigma_{e} / 2$ (see Figure 1(b)).

Further, substituting the first relation (21) in the first equation (18), we obtain the Ginzburg-Landau-Khalatnikov

$$
\dot{m}=m^{\prime \prime}-\frac{\partial E}{\partial m}
$$

which is determined by the energy of vacancy

$$
E(m)=\frac{m^{2}}{2}-\frac{\sigma_{e}}{2} \ln \left[1+\frac{m^{2}}{\gamma^{2}}\right] .
$$

As shown in Figure 2, with $\sigma_{e}<\gamma^{2}$ energy (23) is a monotonically increasing form (curve 1 ), with a minimum of $m_{0}=0$, corresponding to solid state. If the voltage $\sigma_{e}$ exceeds the critical level $\gamma^{2}$ (2, 3 curves in Figure 2), you have at least

$$
m_{0}=\sqrt{\sigma_{e}-\gamma^{2}}
$$

corresponding to the state of plastic flow (see Figure 3).

In this case, substituting the value of (24) to (21), we obtain the final values for the curvature of the velocity of the shear displacement $v_{0}^{\prime}=v^{\prime}\left(m_{0}\right)=m_{0}$ and internal stress $\sigma_{0}=\sigma\left(m_{0}\right)=\gamma^{2}$, corresponding to a given state.

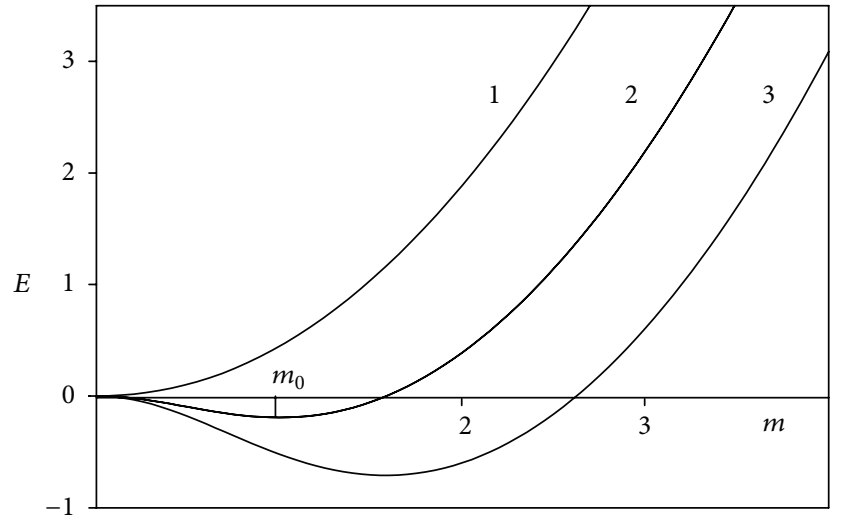

FIgURE 2: The dependence of the vacancy formation energy of its concentration when $1-\sigma_{e}=0.1, \gamma^{2}=0.5 ; 2-\sigma_{e}=2, \gamma^{2}=1$; $3-\sigma_{e}=4, \gamma^{2}=1.5$.

\section{The Stationary Solution}

Consider the solution of (22) in the stationary case, when $\dot{m}=$ 0 . The first integral in this case is given by

$$
E-E_{0}=\frac{\left(m^{\prime}\right)^{2}}{2},
$$

where $E_{0}$ is integration constant. To determine the latter we consider an ordered phase (state of plastic flow) when the concentration of $m=m_{0}$ and $m^{\prime}=0$, respectively. As a result, constant $E_{0}$ represents the energy of the ordered state

$$
E_{0}=E\left(m_{0}\right) \simeq-\frac{1}{4 \gamma^{2}}\left(\sigma_{e}-\gamma^{2}\right)^{2} .
$$

It should be noted that at the boundary of the material $(x \rightarrow \infty)$, which implemented the disordered phase (solid state), the concentration of $m_{\infty}=0$ and thus the vacancy 


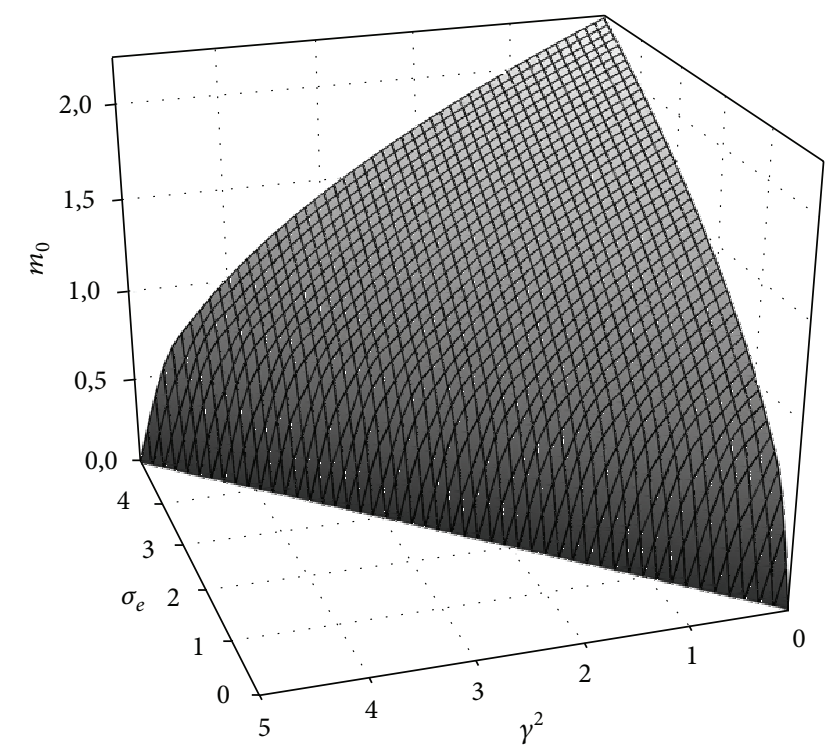

Figure 3: The dependence of the steady-state value of the system parameters $m_{0}$.

formation energy $E=0$, according to (25), lead to a non-zero gradient

$$
m_{\infty}^{\prime}=\frac{m_{0}}{\lambda}, \quad \lambda=\sqrt{\frac{2 \gamma^{2}}{\sigma_{e}-\gamma^{2}}},
$$

where $\lambda$ is characteristic scale, the value of which diverges when the elastic stresses $\sigma_{e}$ critical value $\gamma^{2}$. The presence of the gradient $m_{\infty}^{\prime} \neq 0$ does not mean the appearance of the flow at infinity, since the gradient is due to the disappearance of vacancies $\left(m_{\infty}=0\right)$.

Using the approximation $\sigma_{e} \approx \gamma^{2}$ and given that the rms fluctuations are reduced along the axis $x$, the first derivative we obtain is

$$
m^{\prime}=-\frac{1}{\sqrt{2} \gamma}\left(\left(\sigma_{e}-\gamma^{2}\right)-m^{2}\right) .
$$

The final expression for the concentration of vacancies in the definition (27) for the correlation length and the steadystate value (24) takes the form

$$
m=\frac{m_{0}}{2}\left[1-\operatorname{th} \frac{\left(x-x_{\omega}\right)}{\lambda}\right]
$$

shown in Figure 4 ( $x_{\omega}$ is the constant of integration).

In this case the constant $x_{\omega} \geq \lambda$ determines the width of the border area, in which the concentration of vacancies is reduced to steady state (24). Given the dependence of the elastic stress on the concentration of vacancies (21) and expression (29) to find the spatial distribution of the elastic stresses (see Figure 5), we have

$$
\sigma(x)=\frac{4 \gamma^{2} \sigma_{e}}{4 \gamma^{2}+m_{0}^{2}\left[1-\operatorname{th}\left(\left(x+x_{\omega}\right) / 2\right)\right]^{2}} .
$$

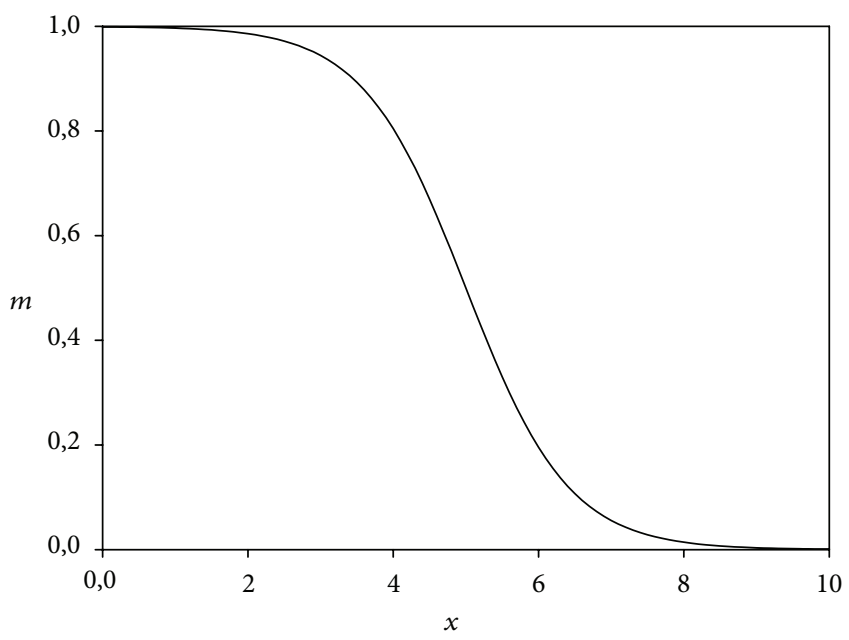

FIGURE 4: The spatial distribution of the concentration of vacancies at $x_{\omega}=5, \gamma^{2}=1$, and $\sigma_{e}=2$.

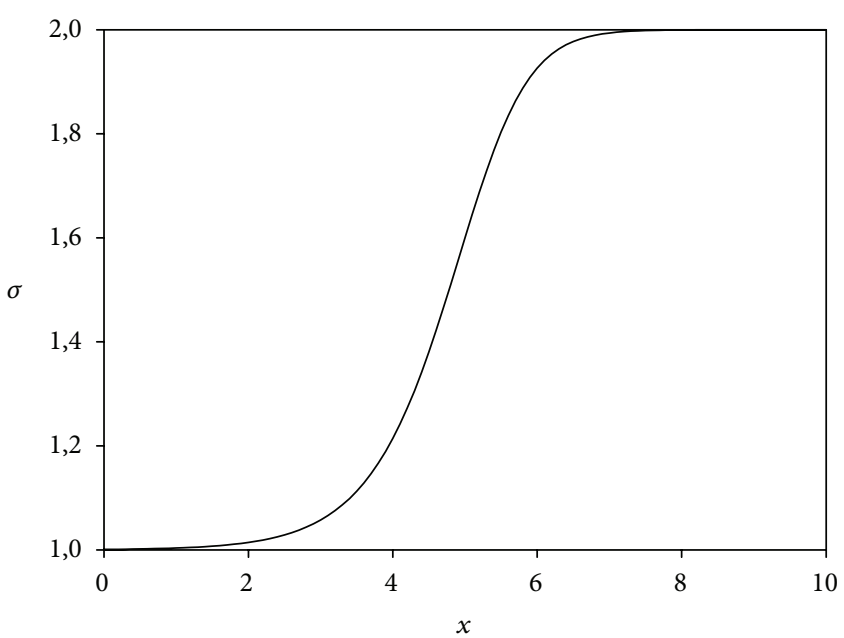

Figure 5: The spatial distribution of the elastic stresses at $x_{\omega}=5$, $\gamma^{2}=1$, and $\sigma_{e}=2$.

As follows from Figure 5, in the transition region elastic stresses increase monotonically from the critical value $\sigma=\gamma^{2}$ to the value $\sigma_{e}$, which is determined by an external influence, which in turn corresponds to increasing the coefficient of friction $\mu=\sigma / P$ values from the hydrodynamic $\eta_{0}^{2} /\left(\bar{\rho} \lambda_{0} t_{m} A G P\right)$ to the value $\sigma_{e} / P$, corresponding to dry friction.

However, in determining the spatial dependence of the shear displacement difficulties, for example, the first equation (21) we can find the first integral

$$
\frac{\sqrt{2} \gamma}{\sigma_{e}} v^{\prime}=-\left(\frac{\sigma_{e}}{\gamma^{2}}\right)^{-1} \ln \frac{1+(m / \gamma)^{2}}{\left(\sigma_{e} /\left(\gamma^{2}-1\right)\right)-(m / \gamma)^{2}},
$$

which has no analytical solution. But the form of the dependence $v(x)$ can be analyzed asymptotically. At the boundary 
of the material, when $x \rightarrow-\infty$, the value of the concentration of vacancies

$$
m \approx m_{0}\left[1-\exp \left(2 \frac{x+x_{\omega}}{\lambda}\right)\right]
$$

gives the spatial dependence

$$
v=\frac{\sigma_{e}}{\sqrt{\sigma_{e}-\gamma^{2}}}\left[\left(\frac{x+x_{\omega}}{\lambda}\right)^{2}-\ln \left(\frac{\sigma_{e}}{2\left(\sigma_{e}-\gamma^{2}\right)}\right)\left(\frac{x+x_{\omega}}{\lambda}\right)\right] .
$$

When $x \rightarrow+\infty$, the expression for the vacancy concentration and rate of shear displacement, respectively, take the form

$$
\begin{gathered}
m \approx \sqrt{\sigma_{e}-\gamma^{2}} \exp \left(-2 \frac{x+x_{\omega}}{\lambda}\right), \\
v=\frac{\sigma_{e}}{\sqrt{\sigma_{e}-\gamma^{2}}} \ln \left[\gamma^{2}\left(\sigma_{e}-\gamma^{2}\right)\right]\left(\frac{x+x_{\omega}}{\lambda}\right) .
\end{gathered}
$$

\section{Conclusion}

Our analysis shows that the use of the system (11)-(13) allows us to take into account the effect of point defects (vacancies and interstitials) on the self-consistent picture of the transition from a solid state to a state of plastic flow under the action of external loads. In this case, the order parameter characterizing the state of the material is the concentration of vacancies for crystalline solid or the proportion of free local volume for amorphous solids.

In the framework of the adiabatic approximation, it is shown that an increase in the curvature of the vacancy rate of the shear displacement increases and the internal voltage drops below the level fixed by external factors. Obtained from the Ginzburg-Landau-Khalatnikov vacancy formation energy becomes non-zero minimum $m_{0}$, typical of the state of plastic flow, provided that the external voltage $\sigma_{e}$ exceeds a critical level $\gamma^{2}$, determining the parameters of the medium. In addition, it is shown that the steady-state spatial distribution of the concentration of vacancies is a falling form while shearing stress component increases monotonically.

\section{References}

[1] L. D. Landau and E. M. Lifshitz, Theory of Elasticity, Elsevier, Oxford, UK, 1986.

[2] A. Liu and S. R. Nagel, Jamming and Rheology: Constrained Dynamics on Microscopic and Macroscopic Scales, Taylor and Francis, London, UK, 2001.

[3] A. Ikeda, L. Berthier, and P. Sollich, "Unified study of glass and jamming rheology in soft particle systems," Physical Review Letters, vol. 109, Article ID 018301, 2012.

[4] B. Devincre and L. P. Kubin, "Mesoscopic simulations of dislocations and plasticity," Materials Science and Engineering A, vol. 234-236, pp. 8-14, 1997.

[5] V. Bulatov, F. F. Abraham, L. Kubin, B. Devincre, and S. Yip, "Connecting atomistic and mesoscale simulations of crystal plasticity," Nature, vol. 391, no. 6668, pp. 669-672, 1998.
[6] K. W. Schwarz, "Simulation of dislocations on the mesoscopic scale. I. Methods and examples," Journal of Applied Physics, vol. 85, article 108, 1999.

[7] A. Artemev, Y. Jin, and A. G. Khachaturyan, "Threedimensional phase field model of proper martensitic transformation," Acta Materialia, vol. 49, no. 7, pp. 1165-1177, 2001.

[8] A. Onuki, "Plastic flow in two-dimensional solids," Physical Review E, vol. 68, Article ID 061502, 2003.

[9] P. D. Fleming and C. Cohen, "Hydrodynamics of solids," Physical Review B, vol. 13, pp. 500-516, 1976.

[10] C. Cohen, P. D. Fleming, and J. H. Gibbs, "Hydrodynamics of amorphous solids with application to the light-scattering spectrum," Physical Review B, vol. 13, pp. 866-877, 1976.

[11] K. Takae and A. Onuki, "Phase-field model of solid-liquid phase transition with density difference and latent heat in velocity and elastic fields," Physical Review E, vol. 83, Article ID 041504, 2011.

[12] L. D. Landau and E. M. Lifshitz, Fluid Mechanics, Pergamon, New York, NY, USA, 1959.

[13] A. Onuki, "Nonlinear strain theory of plastic flow in solids," Journal of Physics, vol. 15, pp. S891-S901, 2003.

[14] D. Ruelle and F. Takens, "On the nature of turbulence," Communications in Mathematical Physics, vol. 20, no. 3, pp. 167-192, 1971.

[15] H. Haken, Synergetic, Sprinder, Berlin, Germany, 1980.

[16] E. N. Lorenz, "Deterministic nonperiodic flow," Journal of the Atmospheric Sciences, vol. 20, no. 2, pp. 130-141, 1963.

[17] O. V. Yushchenko and A. Y. Badalyan, "Statistical description of the collective motion of nanoparticles," Physical Review E, vol. 85, Article ID 051127, 2012.

[18] A. I. Olemskoi, O. V. Yushchenko, and T. I. Zhilenko, "Investigation of conditions for a self-organized transition to the bistable regime of quasi-equilibrium condensation and stripping of the surface," Physics of the Solid State, vol. 53, no. 4, pp. 845-853, 2011. 

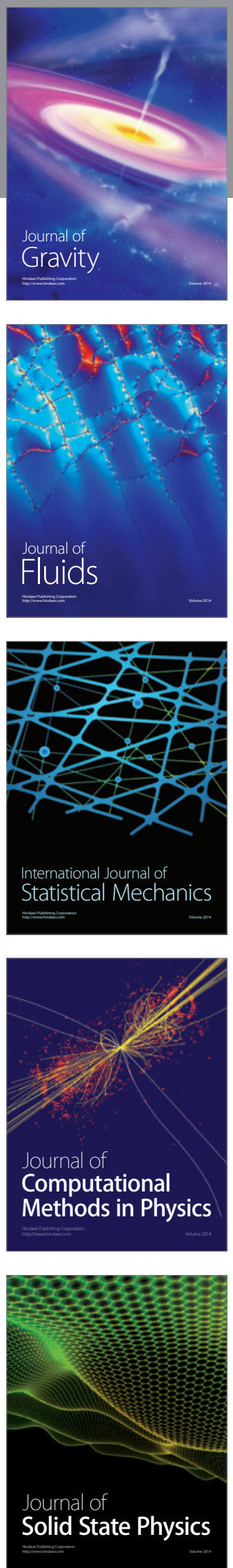

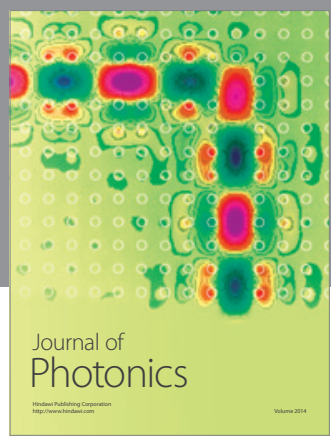

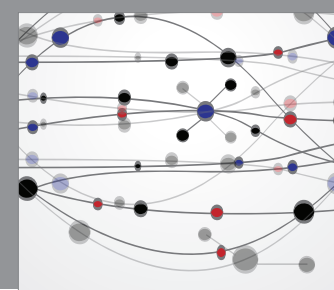

The Scientific World Journal

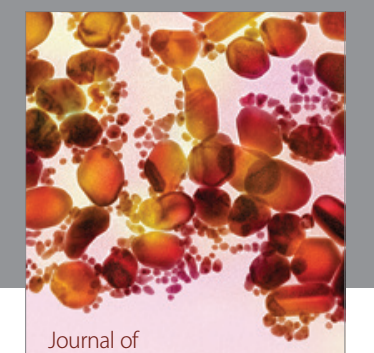

Soft Matter
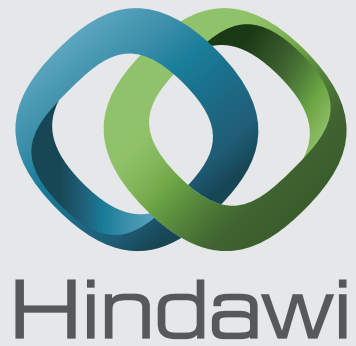

Submit your manuscripts at

http://www.hindawi.com
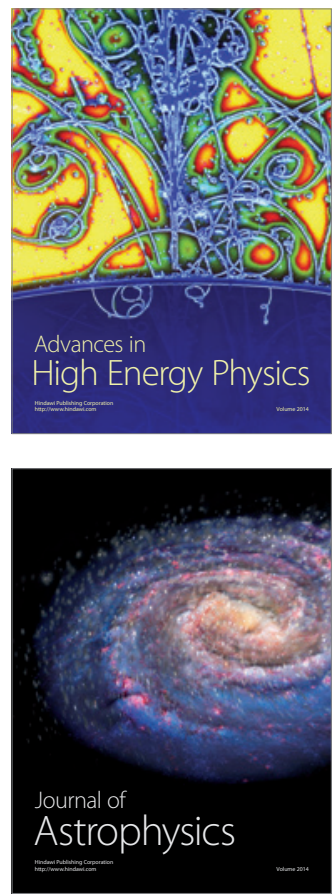
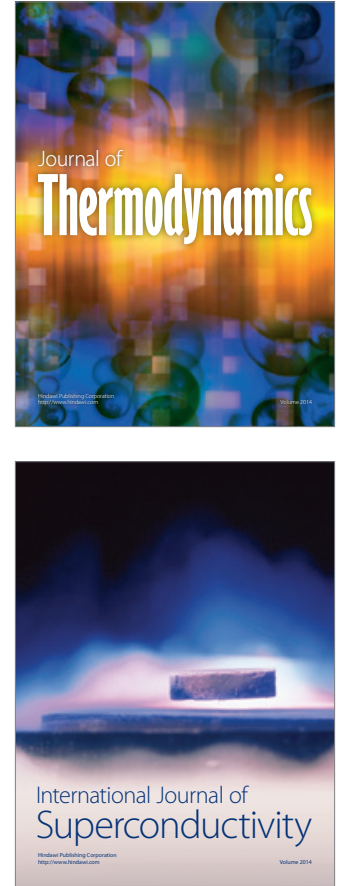
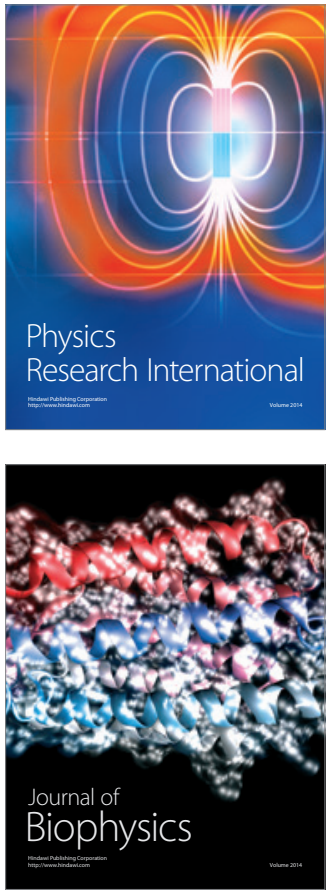
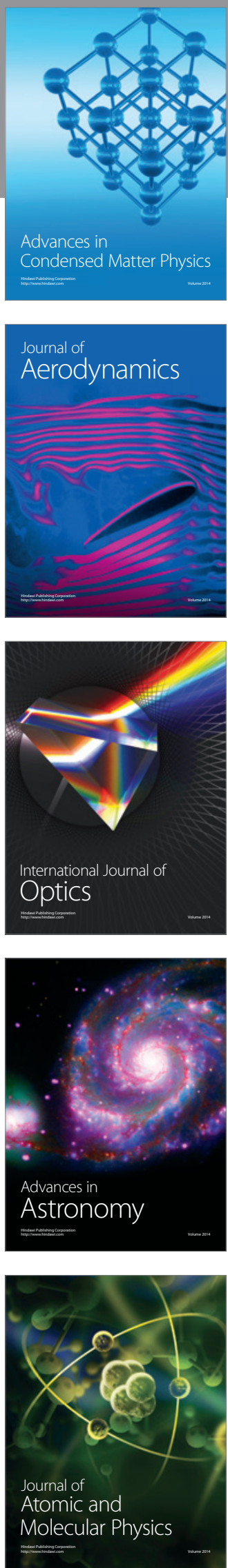